\title{
Studies on the Functional Mechanism of System II Herbicides in Isolated Chloroplasts
}

\author{
G. Renger, R. Hagemann, and W. F. J. Vermaas \\ Max-Volmer-Institut für Biophysikalische und Physikalische Chemie, \\ Technische Universität Berlin, Strasse des 17. Juni 135, D-1000 Berlin 12, \\ Bundesrepublik Deutschland
}

Z. Naturforsch. 39 c, 362-367 (1984); received December 1, 1983

Chloroplasts, Proteolytic Enzymes, Fluorescence, Oxygen Evolution, Herbicide Binding

The effect of specific proteolytic enzymes on variable fluorescence, $p$-benzoquinone-mediated oxygen evolution, PS II herbicide (atrazine and bromoxynil) binding, and protein degradation has been analyzed in isolated class II pea chloroplasts.

It was found that:

1. Trypsin and a lysine-specific protease effectively reduce the maximum chlorophyll- $a$ fluorescence yield, whereas the initial fluorescence remains almost constant. At the same number of enzymatic activity units both proteases have practically the same effect.

2. Trypsin and a lysine-specific protease inhibit the $p$-benzoquinone-mediated flash-induced oxygen evolution with trypsin being markedly more effective at the same number of activity units of both enzymes. Unstacked thylakoids exhibit a higher sensitivity to proteolytic degradation by both enzymes.

3. Trypsin and a lysine-specific protease reduce the binding capacity of $\left[{ }^{14} \mathrm{C}\right]$ atrazine, but enhance that of $\left[{ }^{14} \mathrm{C}\right]$ bromoxynil (at long incubation times trypsin treatment also impairs bromoxynil binding). At the same specific activity a markedly longer treatment is required for the lysine-specific protease in order to achieve the same degree of modification as with trypsin.

4. Trypsin was found to attack the rapidly-turned-over $32 \mathrm{kDa}$-protein severely, whereas the lysine-specific protease does not modify this polypeptide. On the other hand, the lysine-specific protease attacks the light harvesting complex II.

5. Under our experimental conditions an arginine-specific protease did not affect chlorophyll- $a$ fluorescence yield, $p$-benzoquinone-mediated oxygen evolution, herbicide binding and the polypeptide pattern.

Based on these results a mechanism is proposed in which an as yet unidentified polypeptide with exposable lysine residues, as well as the lysine-free " $\mathrm{Q}_{\mathrm{B}}$-protein" regulate the electron transfer from $\mathrm{Q}_{\mathrm{A}}^{-}$to $\mathrm{Q}_{\mathrm{B}}$ and are involved in herbicide binding.

\section{Introduction}

Among the great variety of herbicides interfering with plant metabolism at different stages, products are widely used in modern agriculture which effectively block photosynthetic electron transport between the primary and secondary plastoquinones (referred to as $\mathrm{Q}_{\mathrm{A}}$ and $\mathrm{Q}_{\mathrm{B}}$ ) at the photosystem II acceptor side (for review see [1]). Based upon results obtained in mildly trypsinized chloroplasts, $Q_{A}$ and $\mathrm{Q}_{\mathrm{B}}$ are inferred to be bound to a proteinaceous

Abbreviations: Atrazine, 2-chloro-4-(ethylamino)-6-(isopropylamino)-s-triazine; $p$-BQ, $p$-benzoquinone; bromoxynil, 3,5-dibromo-4-hydroxybenzonitrile; DCMU, 3-(3,4-dichlorophenyl)-1,1-dimethylurea; LHC, light harvesting complex; PS II, photosystem II; $\mathrm{Q}_{\mathrm{A}}$, primary plastoquinone at the photosystem II acceptor side; $\mathrm{Q}_{\mathrm{B}}$, secondary plastoquinone at the photosystem II acceptor side; SN 58132, isopropyl-N-(3-N-ethyl-N-phenyl-carbamoyloxyphenyl)carbamate; SDS/PAGE, sodium dodecyl sulfate/polyacrylamide gel electrophoresis.

Reprint requests to Prof. G. Renger.

0341-0382/84/0500-0362 \$01.30/0 complex that functions [2]: (a) as regulator for the electron transport between $\mathrm{Q}_{\mathrm{A}}$ and $\mathrm{Q}_{\mathrm{B}}$, (b) as barrier to exogeneous redox components and protons, and (c) as binding area for PS II herbicides that interrupt electron transfer from $\mathrm{Q}_{\overline{\mathrm{A}}}$ to $\mathrm{Q}_{\mathrm{B}}$. Later is was postulated [3] that $\mathrm{Q}_{\mathrm{B}}$ is not a tightly bound specific plastoquinone, but a molecule from the plastoquinone pool which statistically occupies, via noncovalent interactions, a specific binding site. After reduction by $\mathrm{Q}_{\overline{\mathrm{A}}}$ the semiquinone form $\mathrm{Q}_{\overline{\mathrm{B}}}$ becomes tightly bound and stabilized. A second transfer step from $Q_{\bar{A}}$ reduces $Q_{\bar{B}}$ to the quinol, which is released via an exchange reaction with another plastoquinone molecule from the pool. PS II herbicides are assumed to "compete" with plastoquinone for binding to the herbicide/ quinone binding environment. This idea is in line with recent findings of a seemingly competitive interaction between binding of quinones and herbicides [4]. Photoaffinity labeling experiments with different types of PS II herbicides have shown that the "clas- 
sical" inhibitors (DCMU, atrazine) predominantly bind to a $32 \mathrm{kDa}$ polypeptide, whereas phenol-type inhibitors are associated, among others, with 40 to $50 \mathrm{kDa}$ components which may be related to the system II reaction center complex (for recent review see [5]). Therefore, more than one polypeptide seems to participate in creating the environment to which quinones and herbicides bind.

Trypsin treatment was found to interrupt $\mathrm{Q}_{\overline{\mathrm{A}}}$ reoxidation in the same manner as DCMU in normal chloroplasts [6], to decrease the binding of DCMU-type inhibitors [7] and, concomitantly, to increase the accessibility of $\mathrm{Q}_{\overline{\mathrm{A}}}$ to $\mathrm{K}_{3}\left[\mathrm{Fe}(\mathrm{CN})_{6}\right]$ [8]. Recently, a lysine-specific protease was shown to induce a partial recovery of oxygen evolution with $\mathrm{K}_{3}\left[\mathrm{Fe}(\mathrm{CN})_{6}\right]$ as acceptor in chloroplasts inhibited by SN 58132 [9] despite the fact that the $32 \mathrm{kDa}$ polypeptide does not contain any lysine [10]. The effect of the inhibitor ioxynil was not changed. On the other hand, an arginine-specific protease appears to be inefficient [9]. These findings might suggest that the effect of herbicides is modified by a polypeptide that contains surface-exposed lysine residues.

In order to confirm this idea, the effect of specific proteolytic enzymes on chlorophyll- $a$ fluorescence, electron transport, herbicide binding, and polypeptide-degradation patterns have been analyzed. The data presented favor the involvement of, among others, a protein with surface-exposable lysine residues in creating the quinone/herbicide binding environment.

\section{Materials and Methods}

Class II pea chloroplasts were prepared as described in [4]. Mild proteolysis was performed in the dark at room temperature by incubation of chloroplasts $\left(50 \mu \mathrm{g} \cdot \mathrm{ml}^{-1}\right)$ with trypsin or the lysine- or arginine-specific protease of 0.25 activity units per $\mathrm{ml}$ (unless indicated otherwise) in a reaction medium containing $10 \mathrm{mM} \mathrm{NaCl}, 5 \mathrm{~mm} \mathrm{MgCl}_{2}, 0.3 \mathrm{M}$ sorbitol and $50 \mathrm{~mm}$ Tricine- $\mathrm{NaOH}, \mathrm{pH}=7.6$, and, when applicable, the labeled inhibitor $\left(\left[{ }^{14} \mathrm{C}\right]\right.$ atrazine or $\left[{ }^{14} \mathrm{C}\right]$ bromoxynil). Where indicated, $\mathrm{MgCl}_{2}$ was omitted from the reaction medium. The time of incubation with a protease is indicated in the Figure legends.

The chlorophyll a fluorescence measurements were performed as in [9]. For measurements of flash-induced $\mathrm{O}_{2}$-evolution, a Clark-type electrode was used which has been described in [12]. The analysis of the polypeptide degradation pattern by SDS/PAGE was performed as in [11].

The arginine- and lysine-specific proteases were obtained from Boehringer, Mannheim, West-Germany.

\section{Results}

In order to study the functional role of different polypeptides for the electron transfer between $Q_{A}$ and $\mathrm{Q}_{\mathrm{B}}$ and the action of PS II herbicides, modifications were analyzed that are caused by specific enzymes attacking proteins selectively at the level of lysine or arginine or both (in the case of trypsin).

\section{Effects on variable fluorescence}

The variable fluorescence yield, $F_{\mathrm{v}}$, and its modification by cations (as well as grana stacking) was found to be highly sensitive to tryptic digestion [13]. The effects were inferred to be caused by a specific attack on lysine residues near the C-terminal end of the light harvesting complex of system II (LHC II) (the structure of the C-terminus is (Lys or Arg)-SerAla-Thr-Thr-Lys-Lys [14]). Accordingly, trypsin and a lysine-specific protease should exert almost the same effect on the variable fluorescence, while an argininespecific protease could be inactive. The data obtained in pea chloroplasts suspended in the reaction medium containing $5 \mathrm{mM} \mathrm{MgCl}$ are depicted in Fig. 1. At the same specific activity, trypsin and lysine-spe-

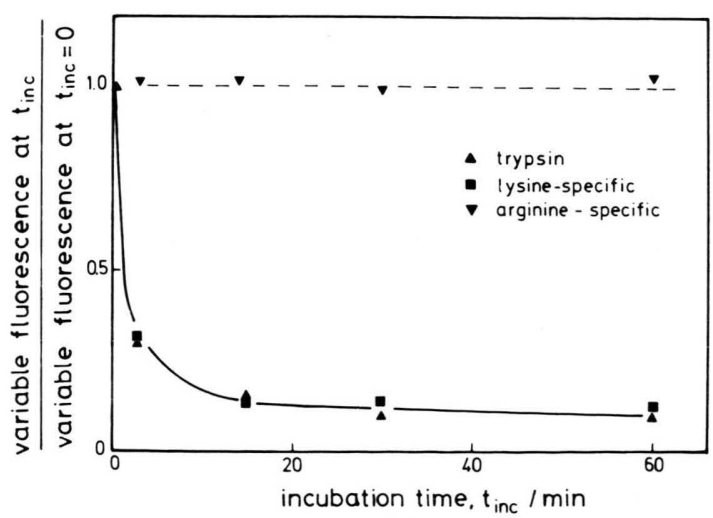

Fig. 1. Variable chlorophyll $a$ fluorescence normalized to the control value (at $t_{\text {inc }}=0$ ) as a function of incubation time, $t_{\text {inc }}$, with proteolytic enzymes in isolated pea chloroplasts. 0.25 units of proteolytic activity per $50 \mu \mathrm{g}$ chlorophyll for trypsin and the lysine-specific protease; 2.5 units for the arginine-specific protease. 
cific protease induce a rapid, almost parallel degradation of the variable fluorescence due to the decrease of the maximum level, $F_{\max }$, whereas the initial level, $F_{0}$, remains nearly unaffected. In contrast to that, at an even tenfold higher activity, the arginine-specific protease does not modify fluorescence emission. As the LHC-II is present mainly in the appressed membrane region, the arginine-specific protease might be prevented from being active simply by steric hindrance. In order to eliminate this effect, chloroplasts were prepared in an $\mathrm{Mg}^{2+}$ free medium and resuspended for the fluorescence measurements in the reaction buffer described in Materials and Methods, but lacking $\mathrm{MgCl}_{2}$. In this case, the variable fluorescence yield is significantly smaller than in the control but with respect to the attack of proteolytic enzymes, the arginine-specific protease was found to be completely inactive (data not shown). Also, this enzyme did not prevent the $\mathrm{Mg}^{2+}$-induced increase of $F_{\max }$ and $F_{\mathrm{v}}$, while trypsin and the lysine-specific protease completely eliminated this effect. These results show that LHC II does not contain surface-exposed arginine residues which can be attacked by proteolytic enzymes.

\section{Effects on PS II electron transport}

The oxygen evolution induced by repetitive flashes was shown to be rather resistent to mild trypsin treatment provided that $\mathrm{K}_{3}\left[\mathrm{Fe}(\mathrm{CN})_{6}\right]$ is added as exogeneous electron acceptor, whereas a rapid decline is observed in the presence of $p$ benzoquinone ( $p$-BQ) [2]. This effect can be reasonably explained assuming that $p$-BQ functions as electron acceptor only if the binding site for endogeneous plastoquinone is intact. Accordingly, the effect of different proteolytic enzymes on the oxygen evolution mediated by $p$-BQ has been measured in stacked and unstacked pea chloroplasts. The data depicted in Fig. 2 reveal that trypsin as well as the lysine-specific protease markedly inhibit the reaction, whereas the arginine-specific protease had no effect, even at a tenfold higher enzymatic activity and regardless of the stacking state of the thylakoid membranes. This shows that the polypeptides required for the acceptor function of $p$-BQ do not contain surface-exposed arginine residues that can be attacked with the specific proteolytic enzyme used in this study.

Compared with the effects on the variable fluorescence, two remarkable differences are observed

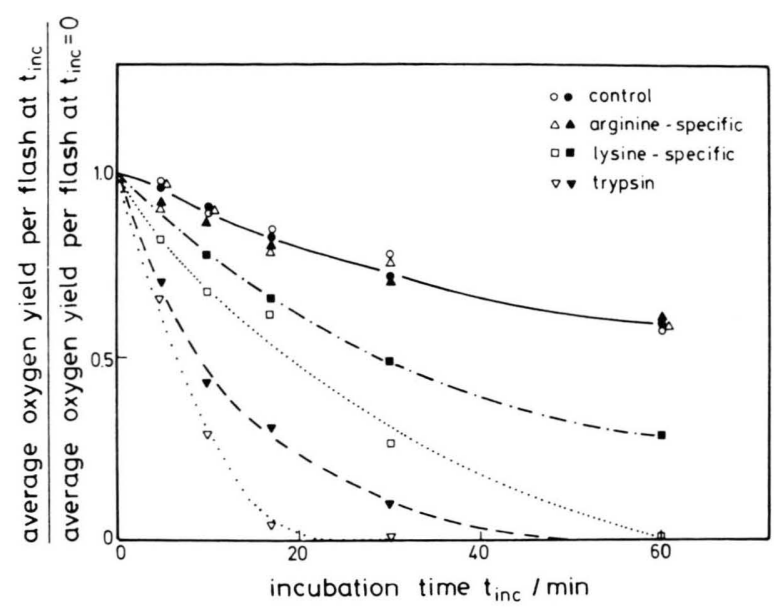

Fig. 2. Average oxygen yield per flash normalized to the control value (at $t_{\text {inc }}=0$ ) as a function of incubation time, $t_{\text {inc }}$, with proteolytic enzymes in isolated pea chloroplasts. $0.1 \mathrm{~mm} p$-BQ served as electron acceptor. The reaction medium was as described in Materials and Methods (closed symbols) or lacked $5 \mathrm{~mm} \mathrm{MgCl}_{2}$ (open symbols). The ratio of the control value of the $\mathrm{O}_{2}$ yield per flash in $\mathrm{Mg}^{2+}$-containing and $\mathrm{Mg}^{2+}$-depleted chloroplasts amounts to ca. 1.5. Addition of proteases as in Fig. 1.

for the action of trypsin and the lysine-specific protease on oxygen evolution with $p$-BQ as electron acceptor: (a) trypsin exhibits a significantly higher activity and (b) the modification by trypsin as well as the lysine-specific protease occur at an increased rate in unstacked compared to stacked thylakoids. Therefore, the results depicted in Fig. 2 are not simply caused by effects of LHC II on the electron transfer to $p$-BQ. Furthermore, a proteolytic cleavage at the $32 \mathrm{kDa}$-herbicide binding polypeptide cannot be responsible for the blockage of electron transport because this protein was reported to be free of lysine [10].

\section{Effects on herbicide binding}

The herbicide binding studies were performed as in [15]. Two compounds were used: ${ }^{14} \mathrm{C}$-labeled atrazine and bromoxynil. The data are depicted in Fig. 3. In the case of atrazine binding, the well-known pattern is observed when using trypsin as proteolytic enzyme. With increasing incubation time the number of herbicide binding sites becomes diminished concomitant with an increase of the dissociation constant, $K_{\mathrm{d}}$. Qualitatively the same effects are observed for the lysine-specific protease. In contrast to that, the arginine-specific protease does not exert 


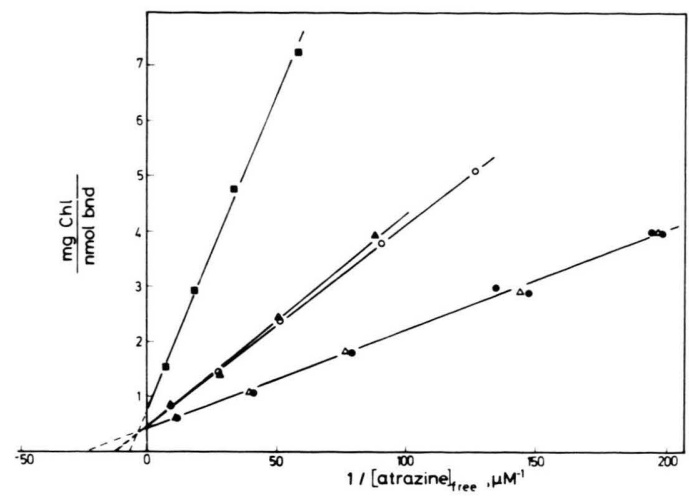

Fig. 3. Double-reciprocal plot for binding of $\left[{ }^{14} \mathrm{C}\right]$ atrazine to pea thylakoids treated with proteolytic enzymes. control; $\triangle, 60 \mathrm{~min}$ arginine-specific protease; $\bigcirc, 60 \mathrm{~min}$ lysine-specific protease; $\boldsymbol{\Delta}, 5$ min trypsin; $\mathbf{\square}, 20$ min trypsin.

any influence, even at a tenfold higher enzymatic activity.

A more complex situation arises for bromoxynil binding. The data shown in Fig. 4 indicate that after a short trypsin-treatment the binding capacity increases due to a tighter attachment $\left(K_{\mathrm{d}}\right.$-decrease) rather than a change in the number of binding sites. The same effect can be achieved with lysine-specific protease. After extensive trypsin treatment the binding capacity of bromoxynil becomes diminished too, but this effect will not be discussed here. As in the case of atrazine binding, the arginine-specific protease was totally ineffective in modifying bromoxynil-binding properties.

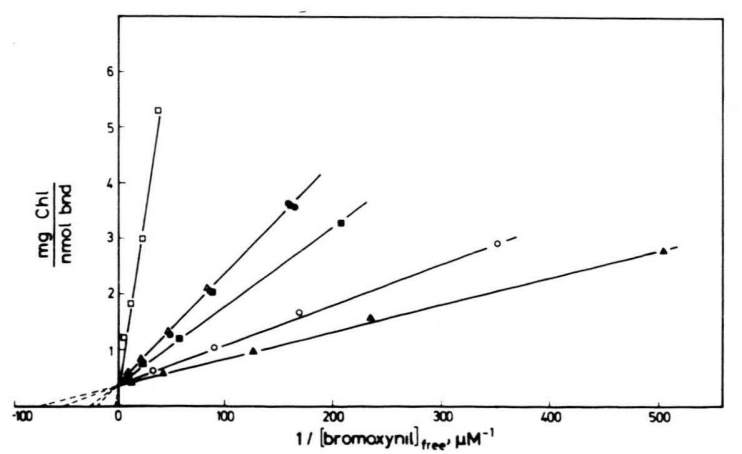

Fig. 4. Double-reciprocal plot for binding of $\left[{ }^{14} \mathrm{C}\right]$ bromoxynil to pea thylakoids treated with proteolytic enzymes. control; $\triangle, 60 \mathrm{~min}$ arginine-specific protease; $\bigcirc, 60 \mathrm{~min}$ lysine-specific protease; $\boldsymbol{A}, 5 \mathrm{~min}$ trypsin; $\mathbf{E}, 20 \mathrm{~min}$ trypsin; $\square, 60$ min trypsin.
The modifications of herbicide binding by proteolytic enzymes reported in Figs. 3 and 4 are not simply caused by an unstacking effect, because $\mathrm{Mg}^{2+}$-depletion did not affect atrazine binding and increased the dissociation constant $K_{\mathrm{d}}$ for bromoxynil binding [16]. It is interesting to note that, in contrast to the fluorescence yield data but in qualitative correspondence with the $p$-BQ-mediated oxygen evolution, trypsin exhibits a significantly faster modification of the binding properties compared to the effect of the lysine-specific protease.

\section{Effects on the polypeptide pattern}

The effects of the proteolytic enzymes on $p$-BQmediated oxygen evolution and on the herbicide binding capacity suggest that surface-exposed lysine residues play an essential role in the structural integrity of polypeptide(s) that affect electron transport and interact with herbicides at the acceptor side of system II. On the other hand, atrazine is claimed to be a prototype for herbicides which specifically bind to a $32 \mathrm{kDa}$-polypeptide $[17,18]$ that was reported to be free of lysine [10]. In order to be able to draw a reasonable conclusion, it remains to be proven that the lysine-specific protease actually does not affect the $32 \mathrm{kDa}$-herbicide binding protein. Therefore, comparative measurements were performed for analysis of the proteolytic cleavage pattern of trypsin and the lysine-specific protease (for the sake of completeness the effect of the arginine-specific protease was also measured).

Pea chloroplasts isolated from plants grown for 3 hours in the presence of $\left[{ }^{35} \mathrm{~S}\right]$ methionine were used, because the $32 \mathrm{kDa}$-atrazine binding polypeptide is characterized by a very rapid turnover so that a rather specific labeling can be achieved during the comparatively short $\left[{ }^{35} \mathrm{~S}\right]$ methionine supply. Under these conditions, LHC II becomes significantly labeled too, due to the relatively large amount of this protein in the thylakoids. After thylakoid isolation, proteolytic treatment was carried out and the ${ }^{35} \mathrm{~S}$-labeling of proteins was monitored autoradiographically. The results obtained are depicted in Fig. 5. They reveal a remarkable difference in effects caused by trypsin and lysine-specific protease. In the former case, the $32 \mathrm{kDa}$ peak, reflecting the lysine-free protein, becomes drastically reduced compared to other polypeptides of the control pattern, whereas in the latter 


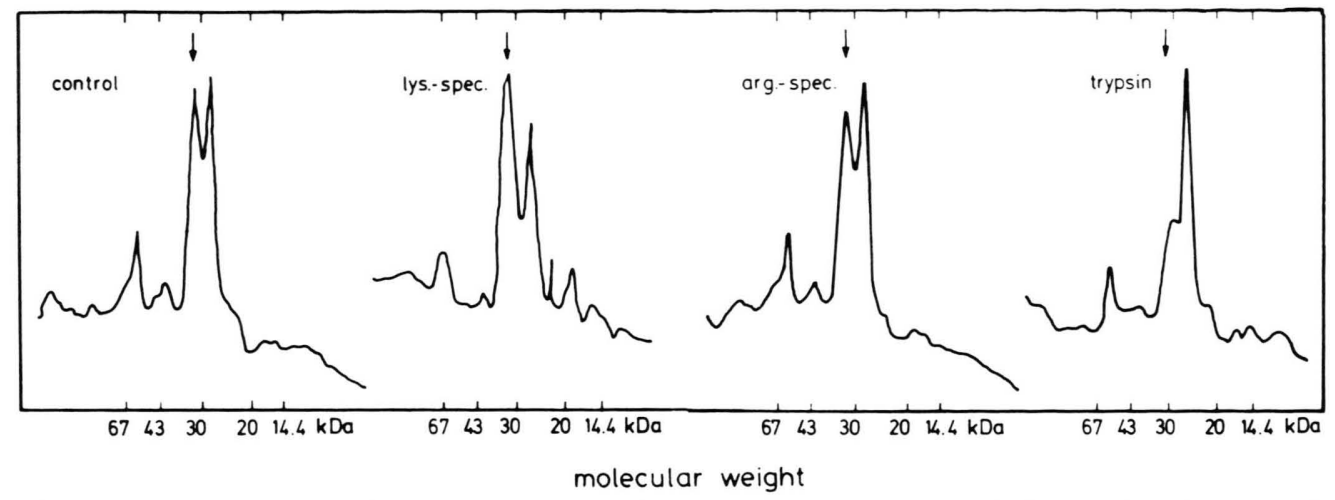

Fig. 5. Densitometer scan of an autoradiogram of a SDS/PAGE gel of thylakoid proteins from pea leaves labeled with $\left[{ }^{35} \mathrm{~S}\right]$ methionine for $3 \mathrm{hrs}$ in the light. The isolated thylakoids $\left(50 \mu \mathrm{g} \cdot \mathrm{ml}^{-1}\right)$ were treated with proteolytic enzymes for one hour in the dark as indicated in the Figure. The molecular weights on the abscissae correspond to the position of the marker proteins as determined by Coomassie Brilliant Blue staining. Arrows indicate the position of the apparent molecular weight of $32 \mathrm{kDa}$. Activity of the proteolytic enzymes: arginine-specific, 2.5 units per ml; lysine-specific, 2.5 units per $\mathrm{ml}$; trypsin, 0.025 units per $\mathrm{ml}$.

case almost no effect is observed. These data furnish convincing evidence that the rapidly-turned-over $32 \mathrm{kDa}$-protein is resistent to proteolytic attack by the lysine-specific enzyme.

\section{Discussion}

Most of the current models on the functional mechanism of PS II-herbicide action are based on the assumption that only one herbicide or quinone molecule can be bound to the herbicide/quinone binding environment at a time ("competitive" herbicide/quinone interaction) [3]. The binding interaction between different types of herbicides and quinones may be allosteric (binding of one ligand strongly decreases the affinity of another ligand binding to a non-overlapping site) rather than really competitive [16]. Whatever the precise mechanism of herbicide binding and inhibition of $\mathrm{Q}_{\mathrm{A}}^{-}$oxidation may be, the data shown here imply that not only the $32000 \mathrm{M}_{\mathrm{r}}$ lysine-free protein (" $\mathrm{Q}_{\mathrm{B}}$-protein") is involved in binding herbicides and $\mathrm{Q}_{\mathrm{B}}$, but that also (a) protein(s) with (probably surface-exposed) lysine residues participate(s) in creating the herbicide/quinone binding environment: the lysine-specific protease is able to inhibit $p$ - $\mathrm{BQ}$ reduction as well as to modify herbicide binding (Figs. 2-4).

One might speculate that a modification of LHCII, which is present in relatively high amounts in the thylakoid membrane, could induce structural changes in the PS II environment leading to a modi- fication of electron transfer to $\mathrm{Q}_{B}$ and of the herbicide binding. However, the LHC-II, which is attacked readily by the lysine-specific protease, appears not to be involved in the protease-induced changes in $\mathrm{Q}_{B}$ and herbicide binding because there is no good kinetic parallelism between the decline in variable fluorescence yield, reflecting LHC-II modification (Fig. 1), and inhibition of $p$-BQ reduction (Fig. 2). Furthermore, bromoxynil affinity decreases upon unstacking, whereas atrazine binding is not affected [16]; the effects induced by the lysine-specific protease are very different. Therefore, it is more plausible that the lysine-specific protease modifies a proteinaceous component at the PS II acceptor side different from the lysine-free $32000 \mathrm{M}_{\mathrm{r}}$ protein. Since the $\mathrm{Q}_{\overline{\mathrm{A}}}$ oxidation by $\mathrm{K}_{3}\left[\mathrm{Fe}(\mathrm{CN})_{6}\right]$ is enhanced by the lysine-specific protease [9], it could be attractive to speculate that the protein to which $\mathrm{Q}_{\mathrm{A}}$ is bound (probably the PS II reaction center protein (H. Y. Nakatani, personal communication)) might be the target for the lysine-specific protease inducing the effects on binding of $\mathrm{Q}_{\mathrm{B}}$ and herbicides. However, as the blockage by ioxynil is not prevented by lysine-specific in contrast to trypsin proteolysis, a simple "unshielding" effect of $Q_{A}$ does not explain the data [9], unless ioxynil binding prevents, by conformational changes in the binding environment, an attack by the lysine-specific protease.

One might argue at this stage that the lysine-containing rather than the $32000 \mathrm{M}_{\mathrm{r}}$ lysine-free protein 
is the major regulatory protein of herbicide and quinone binding. However, this is grossly oversimplified. One amino acid in the chloroplast-encoded $32000 \mathrm{M}_{\mathrm{r}}$ lysine-free "Q $\mathrm{Q}_{\mathrm{B}}$-protein" appears to be changed in (maternally inherited) triazine-resistant biotypes (L. McIntosh, personal communication), which results in a dramatically changed affinity for most PS II herbicides [19] and a shifted semiquinone equilibrium between $\mathrm{Q}_{A}$ and $\mathrm{Q}_{B}$ [15]. Therefore, we conclude that the herbicide/quinone binding environment is created by more than one polypeptide: the lysine-free $32000 \mathrm{M}_{\mathrm{r}}$ "Q $\mathrm{Q}_{\mathrm{B}}$-protein" and a lysine-containing polypeptide are major components. Changes in one of the polypeptides induce a modification of the properties of the herbicide/quinone binding environment (directly or indirectly

[1] C. Fedtke, Biochemistry and Physiology of Herbicide Action, Springer Verlag, Berlin 1982.

[2] G. Renger, Biochim. Biophys. Acta 440, 287-300 (1976).

[3] B. R. Velthuys, FEBS Lett. 126, 277-281 (1981).

[4] W. F. J. Vermaas, C. J. Arntzen, L.-Q. Gu, and C. A. Yu, Biochim. Biophys. Acta 723, 266-275 (1983).

[5] U. Johanningmeier, E. Neumann, and W. Oettmeier, J. Bioenerg. Biomembr. 15, 43-66 (1983).

[6] G. Renger and W. Weiss, FEBS Lett. 137, 217-221 (1982).

[7] W. Tischer and H. Strotmann, Z. Naturforsch. 34c, 992-995 (1979).

[8] G. Renger, FEBS Lett. 69, 225-230 (1976).

[9] G. Renger, R. Hagemann, and G. Dohnt, Photobiochem. Photobiophys. 5, 273-279 (1983).

[10] G. Zurawski, H. J. Bohnert, P. R. Whitfeld, and W. Bottomley, Proc. Natl. Acad. Sci. USA 79, 7699 to 7703 (1982).

[11] R. Hagemann, W. F. J. Vermaas, and G. Renger, Advances in Photosynthesis Research (C. Sybesma, ed.), Vol. 4, pp. 17-20, Martinus Nijhoff/Dr. W. Junk Publishers, Den Haag 1984. (allosterically)) resulting in a change in the binding characteristics of many ligands to the environment.

\section{Acknowledgements}

The authors would like to thank Dipl.-Chem. A. Schulze for performing the fluorescence measurements, Dipl.-Phys. G. Dohnt for a critical reading of the manuscript and S. Hohm-Veit for skillful technical assistance. We thank May and Baker, Ltd. (England) and CIBA-GEIGY (Greensboro, NC, USA) for the generous gifts of $\left[{ }^{14} \mathrm{C}\right]$ bromoxynil and $\left[{ }^{14} \mathrm{C}\right]$ atrazine, respectively. The financial support by the Bundesministerium für Forschung und Technologie (KBF 46), Deutscher Akademischer Austauschdienst and Deutsche Forschungsgemeinschaft is gratefully acknowledged.

[12] G. Renger, Biochim. Biophys. Acta 256, 428-439 (1972).

[13] K. E. Steinback, J. J. Burke, and C. J. Arntzen, Arch. Biochem. Biophys. 195, 546- 557 (1979).

[14] J. E. Mullet, T. O. Baldwin, and C. J. Arntzen, Proc. 5 th Int. Congr. Photosynth. (G. Akoyonoglou, ed.), Vol. III, pp. 577-582, Balaban Int. Sci. Serv., Philadelphia 1981.

[15] W. F. J. Vermaas, Ph.D. Thesis, Agricultural University, Wageningen, The Netherlands (1984).

[16] W. F. J. Vermaas, G. Renger, and C. J. Arntzen, Z. Naturforsch. 39 c, 368-373 (1984).

[17] K. Pfister, K. E. Steinback, G. Gardner, and C. J. Arntzen, Proc. Natl. Acad. Sci. USA 78, 981-985 (1981).

[18] G. Gardner, Science 211, $937-940$ (1981).

[19] K. Pfister and C. J. Arntzen, Z. Naturforsch. 34c, 996- 1009 (1979). 